\title{
Symmetry-adapted non-equilibrium molecular dynamics of chiral carbon nanotubes under tensile loading
}

\author{
Amin Aghaei $^{\mathrm{a})}$ and Kaushik Dayal ${ }^{\mathrm{b})}$ \\ Civil and Environmental Engineering, Carnegie Mellon University, Pittsburgh, Pennsylvania 15213, USA
}

(Received 6 April 2011; accepted 30 April 2011; published online 16 June 2011)

\begin{abstract}
We report on non-equilibrium molecular dynamics calculations of chiral single-wall carbon nanotubes using the framework of Objective Structures. This enables us to adapt molecular dynamics to the symmetry of chiral nanotubes and efficiently simulate these systems with small unit cells. We outline the method and the adaptation of a conventional thermostat and barostat to this setting. We then apply the method in order to examine the behavior of nanotubes with various chiralities subject to a constant extensional strain rate. We examine the effects of temperature, strain rate, and pre-compression/pretension. We find a range of failure mechanisms, including the formation of Stone-Wales defects, the opening of voids, and the motion of atoms out of the cross-section. (c) 2011 American Institute of Physics. [doi:10.1063/1.3596827]
\end{abstract}

\section{INTRODUCTION}

Carbon nanotubes have a range of outstanding mechanical properties. ${ }^{1}$ They are key candidates for a variety of applications involving both static and dynamic mechanical loads. ${ }^{2}$ In dynamic settings, nanotubes are subject to large strain rates. Hence, it is important to understand the microscopic atomic-level mechanisms that govern their behavior. Such understanding can provide fundamental insights and enable the formulation of predictive mesoscale models. ${ }^{3,4}$

Molecular dynamics (MD) is a key tool for understanding the atomic-level mechanisms of the deformation of carbon nanotubes. However, the large number of atoms in a nanotube requires the simulation of only a small subset of the entire system to be computationally feasible. Whereas crystals have a natural translational symmetry that enables the application of periodic boundary conditions to a small number of atoms, the translational symmetry in nanotubes can require the simulation of a large number of atoms. Hence we apply the framework of Objective Structures (OS).$^{5}$ OS provides a symmetry-adapted approach to the modeling of complex nanostructures. In the case of chiral nanotubes, it provides a generalization of periodic boundary conditions that takes into account both translational and rotational symmetries simultaneously. The resulting Objective boundary conditions enable the simulation of chiral nanotubes with a small set of atoms. In addition, OS provides a method to apply bending ${ }^{6}$ and torsional loads to nanotubes, ${ }^{7}$ as well as non-equilibrium extensional strain rates; ${ }^{8}$ it has been proved that in these methods, each atom in the system obeys exactly the equations of MD without any spurious forces.

This paper makes two specific contributions. The first involves the development of OS methodology. As currently formulated, OS generalizes periodic boundary conditions. An extremely useful tool in equilibrium periodic molecular

\footnotetext{
${ }^{\text {a) }}$ Electronic mail: aghaei@cmu.edu.

b) Author to whom correspondence should be addressed. Electronic mail: kaushik@cmu.edu.
}

dynamics is the use of thermostats ${ }^{9}$ to simulate the effect of a heat bath and barostats ${ }^{10}$ to simulate the effect of an applied far-field stress. These enable MD calculations of systems with specified temperature and stress rather than specified energy and shape. Based on the Berendsen barostat, we present a generalization to the equilibrium OS setting; in particular, we obtain a formula to apply a given axial force along the nanotubes rather than specify the strain rate as previously done. ${ }^{8}$ Additionally, we show that the use of these thermostats and barostats in the appropriate manner does not affect the fundamental property of OS, namely, that applying the equations of MD to the simulated atom automatically implies that all images also satisfy the equation of MD with no fictitious forces.

We then apply OS to a systematic study of failure mechanisms in carbon nanotubes under imposed strain rates. We use the Tersoff potential to model atomic interactions. ${ }^{11}$ Although the mechanics of carbon nanotubes have been extensively studied, previous works have usually been restricted in some important ways. For instance, the extremely large unit cell required for translational periodicity forces the consideration of special chiralities such as armchair and zigzag (see, e.g., Ref. 12). Alternately, periodicity in the axial direction is replaced by fixed-end boundary conditions that can lead to boundary effects. Although multiscale methods that couple finite elements to atoms are useful when applying complex boundary conditions, they are typically valid only for zero temperature and static equilibrium. ${ }^{13}$ At finite temperature, failure mechanisms have been studied using transition state theory; ${ }^{14}$ however, it is not clear that the theory is valid this far away from equilibrium. Although important conclusions regarding the behavior of nanotubes can be obtained, such as the formation and motion of defects, ${ }^{15-17}$ these insights are restricted, particularly in terms of nanotube chirality. In this paper, we calculate the dynamic response of carbon nanotubes over a range of strain rates from $10^{8} \mathrm{~s}^{-1}$ to $10^{4} \mathrm{~s}^{-1}$ and a range of temperatures. We exploit OS in order to explore a large range of chiralities as well as to test against the choice of fundamental domain (FD; the 
OS analog of a unit cell in periodic MD). We also apply the OS analog of a barostat in order to examine the effects of pre-tension and pre-compression on the failure response. We emphasize that barostats and thermostats are applicable only at equilibrium, as in the case of standard MD, and hence we use them to prepare the nanotube before applying non-equilibrium loads.

Our findings are, broadly, that there are three failure mechanisms: brittle-like fracture, bond rotations leading to Stone-Wales (SW) defects, ${ }^{18}$ and bond distortion caused by the motion of a few atoms off the surface in the radial direction. The particular failure mechanism depends on the temperature, strain rate, imposed strain, and chirality. In Refs. 15 and 19-22, it was observed that the Stone-Wales mechanisms have a larger activation barrier and are thus likely at higher temperatures, whereas brittle fracture and out-of-plane bond distortions are likely at low temperatures. The initiation of brittle fracture and out-of-plane bond distortion is followed by an increase in the temperature, and increases the possibility of the nucleation of SW defects. Thus, there is often a mix of failure mechanisms that are responsible for eventual failure.

The paper is organized as follows. In Sec. II, we provide an overview of the OS method. In Sec. III, we extend the formulation of thermostats and barostats to OS. In Sec. IV, we report on the results of our non-equilibrium calculations on single-wall carbon nanotubes. We focus particularly on the influence of strain-rate, chirality, temperature, and pre-compression on the mechanism of failure. Finally, we conclude in Sec. V.

\section{SINGLE-WALLED CARBON NANOTUBES AS OBJECTIVE STRUCTURES}

An objective atomic structure is an atomic assembly in which all atoms see the same environment up to translation and rotation. ${ }^{5}$ Similarly, an objective molecular structure is a structure composed of groups of atoms, with each group forming a "molecule," such that each molecule sees the same environment up to translation and rotation. Thus, an objective atomic structure is a special case of an objective molecular structure with the molecule consisting of a single atom.

A compact and intuitive description of OS is provided by the language of group theory. ${ }^{8,23}$ A discrete group $G$ of isometries in 3D is assumed to consist of elements of the form $g=(\boldsymbol{Q} \mid \boldsymbol{c}), \boldsymbol{Q} \in O(3), \quad$ and $\quad \boldsymbol{c} \in \mathbb{R}^{3}$. Given that $g_{1}=\left(\boldsymbol{Q}_{1} \mid \boldsymbol{c}_{1}\right)$ and $g_{2}=\left(\boldsymbol{Q}_{2} \mid \boldsymbol{c}_{2}\right)$, the rule for group multiplication is $g_{1} g_{2}=\left(\boldsymbol{Q}_{1} \boldsymbol{Q}_{2} \mid \boldsymbol{Q}_{1} \boldsymbol{c}_{2}+\boldsymbol{c}_{1}\right)$, and the rule for inverses is $g^{-1}=\left(\boldsymbol{Q}^{T} \mid-\boldsymbol{Q}^{T} \boldsymbol{c}\right)$. These rules come from the action of these isometries on $\mathbb{R}^{3}: g(\boldsymbol{x})=\boldsymbol{Q x}+\boldsymbol{c}$. Then composition of mappings gives

$$
\begin{aligned}
g_{1}\left(g_{2}(\boldsymbol{x})\right) & =\boldsymbol{Q}_{1}\left(\boldsymbol{Q}_{2} \boldsymbol{x}+\boldsymbol{c}_{2}\right)+\boldsymbol{c}_{1} \\
& =\boldsymbol{Q}_{1} \boldsymbol{Q}_{2} \boldsymbol{x}+\boldsymbol{Q}_{1} \boldsymbol{c}_{2}+\boldsymbol{c}_{1} \\
& =g_{1} g_{2}(\boldsymbol{x}),
\end{aligned}
$$

from which one can infer the rules for products and inverses given above. The identity is $i d=(\boldsymbol{I} \mid 0)$.

Now select any molecule in the OS as the 0th molecule and define this as the fundamental domain of the OS; this is analogous to the unit cell in standard periodic MD. Let the $k$ th atom in the FD have the position $x_{0, k}$ and the atomic mass $m_{k}$, with $k=1, \ldots, M$, and $M$ being the number of atoms per molecule. Given an isometry group $G=\left\{g_{0}:=i d, g_{1}, \ldots, g_{N-1}\right\}, g_{i}=\left(\boldsymbol{Q}_{i} \mid \boldsymbol{c}_{i}\right)$, with $N$ being the number of molecules and possibly infinite, we can construct the OS using

$$
\boldsymbol{x}_{i, k}=g_{i}\left(x_{0, k}\right)=Q_{i} x_{0, k}+\boldsymbol{c}_{i}, \quad g_{i} \in G, \quad k=1,2, \ldots M .
$$

We specialize this for single-wall carbon nanotubes summarizing Ref. 8. Consider a nanotube with a chiral vector described by $(n, m)$ and a nanotube axis $\boldsymbol{e}$. Define the generators $h_{1}, h_{2}$ of this nanotube as isometries of the following form:

$$
\begin{aligned}
& h_{1}=\left(R_{\theta_{1}} \mid 0\right), \quad R_{\theta_{1}} \boldsymbol{e}=\boldsymbol{e}, \quad 0<\theta_{1}=\frac{2 \pi \min (|p|,|q|)}{G C D(n, m)} \leq 2 \pi \\
& h_{2}=\left(R_{\theta_{2}} \mid \kappa_{2} \boldsymbol{e}\right), \quad R_{\theta_{2}} \boldsymbol{e}=\boldsymbol{e}, \\
& \theta_{2}=\pi \frac{p(2 n+m)+q(n+2 m)}{n^{2}+m^{2}+n m}, \quad \kappa_{2}=\frac{3 l_{0}}{2 \sqrt{n^{2}+m^{2}+n m}} .
\end{aligned}
$$

The generator $h_{1}$ is a rotation operation with an axis coinciding with $\boldsymbol{e}$, and $h_{2}$ is a screw operation with the orthogonal part having an axis coinciding with $\boldsymbol{e}$. The quantity $l_{0}=0.142 \mathrm{~nm}$ is the bond length of the graphite sheet before rolling. The integers $p$ and $q$ are such that $p m_{1}-q n_{1}=1$, where $m_{1}=m / \operatorname{GCD}(n, m)$ and $n_{1}=n / \operatorname{GCD}(n, m)$, and $\operatorname{GCD}(n, m)$ is the greatest common divisor of $n$ and $m$.

Now consider the isometry group $G=\left\{h_{1}^{i_{1}} h_{2}^{i_{2}} ; i_{1}, i_{2}\right.$ $\in \mathbb{Z}\}$ generated by the generators $h_{1}, h_{2}$. As shown in Ref. 8, $G$ provides an isometry group to generate the nanotube, and Eq. (2) can be written as

$$
\boldsymbol{x}_{\left(i_{1}, i_{2}\right), k}=h_{1}^{i_{1}} h_{2}^{i_{2}}\left(\boldsymbol{x}_{(0,0), k}\right), \quad i_{1}, i_{2} \in \mathbb{Z} ; k=1,2 .
$$

In this description, the FD contains two carbon atoms, and hence $k=1,2$.

The 2-atom FD above greatly limits the possible atomic motions for the calculations that we wish to perform. It is roughly analogous to a periodic MD calculation with 2 atoms in the unit cell; that would constrain motions to such an extent that defects such as vacancies and dislocations would be unable to form. Therefore, we increase the number of atoms in the FD in order to balance computational efficiency and be able to capture phenomena of interest. To do this, we use a FD with atoms at initial positions given by $h_{1}^{i} h_{2}^{j}\left(\boldsymbol{x}_{(0,0), k}\right)$, where $k=1,2$ and the indices $i, j$ run over $i_{1} \leq i \leq i_{2}, j_{1} \leq j \leq j_{2}$. Then the remainder of the nanotube is obtained from a subgroup $\hat{G}=\left\{h_{1}^{i^{\prime}} h_{2}^{j^{\prime}} ; i^{\prime}, j^{\prime} \in \mathbb{Z}\right\}$, where $\hat{h}_{1}, \hat{h}_{2}$ are the generators of $\hat{G}$. Each element of $\hat{G}$ maps the enlarged FD to an image to form the nanotube:

$$
\begin{aligned}
& \boldsymbol{x}_{\left(i^{\prime}, j^{\prime}\right), k}=\hat{h}_{1}^{i^{\prime}} \hat{h}_{2}^{j^{\prime}}\left(\boldsymbol{x}_{(0,0), k}\right), \\
& i^{\prime}, j^{\prime} \in \mathbb{Z} ; k \in\left\{1,2, \ldots 2 \times\left(i_{1}-i_{2}+1\right) \times\left(j_{1}-j_{2}+1\right)\right\} .
\end{aligned}
$$

The generators $\hat{h}_{1}=h_{1}^{i_{2}-i_{1}+1}, \hat{h}_{2}=h_{2}^{j_{2}-j_{1}+1}$, and $k$ indexes the $2 \times\left(i_{1}-i_{2}+1\right) \times\left(j_{1}-j_{2}+1\right)$ atoms in the enlarged FD. Two examples of FDs that can be generated from such a 


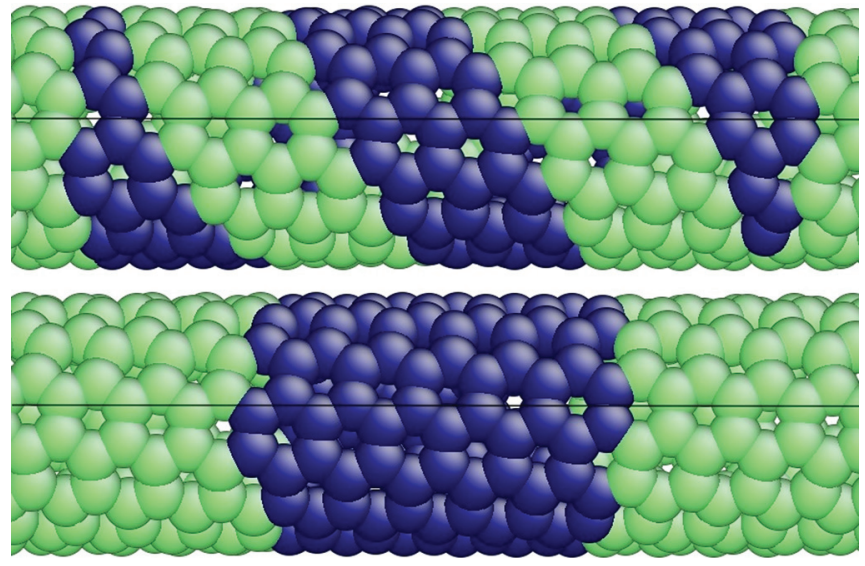

FIG. 1. (Color online) Two fundamental domains with 164 atoms for an $(8,6)$ nanotube. Top: a helical FD with $i_{1}=0, i_{2}=0, j_{1}=0$, and $j_{2}=81$. Bottom: a cylindrical FD with $i_{1}=0, i_{2}=1, j_{1}=0$, and $j_{2}=40$. Dark atoms (blue in online color) belong to the FD, and light atoms (green in online color) belong to the images under the subgroup.

procedure are shown in Fig. 1. We note that this is closely analogous to the use of supercells in periodic MD.

We note certain features of the OS isometry group approach. First, OS above enables facile MD calculations of nanotubes that are chiral, whereas periodic boundary conditions would require extremely long unit cells. Further, periodicity would constrain the deformation and possibly suppress certain failure modes; although OS also does this due to the choice of FD, extremely different FDs can be chosen (e.g., Figure 1) in order to test for artifacts of this choice. Furthermore, OS enables a transparent approach to the application of external loads that cause twisting and extension; the choices for $h_{1}, h_{2}$ can be thought of as corresponding to an unloaded nanotube, and changing $\theta_{2}$ would correspond to imposed twist, whereas changing $\kappa_{2}$ would correspond to imposed extension. Finally, imposed twist can cause a complete lack of translational symmetry in the nanotube and would require, in principle, infinitely long unit cells with periodic $\mathrm{MD}$, but it can be easily and efficiently handled with OS.

\section{OBJECTIVE MD AT CONSTANT TEMPERATURE AND AXIAL FORCE}

Objective molecular dynamics is a generalization of periodic MD (Ref. 7) that uses objective images, rather than periodic ones, to compute the forces on atoms. In Ref. 8, it is shown that one could satisfy the equations of MD even if the elements of the OS isometry group were certain explicit functions of time. For each element $g=(\boldsymbol{Q}(t) \mid \boldsymbol{c}(t))$, the explicit time dependence is restricted to be such that $\boldsymbol{Q}$ is independent of time and $\boldsymbol{c}$ is at most an affine function of time, $\boldsymbol{c}(t):=\boldsymbol{a}+\boldsymbol{t} \boldsymbol{b}$. In addition, the time-dependence of the different elements of the group must be such that they continue to form a group at every time $t$.

Specializing this to the nanotube, if the generator $h_{2}=\left(\boldsymbol{R}_{\theta_{2}} \mid \kappa_{2}(\boldsymbol{t}) \boldsymbol{e}\right)$ has time-dependence such that $\kappa_{2}(t)$ $=a t+b$ with constants $a$ and $b$ and $\boldsymbol{R}_{\theta_{2}}$ independent of time, then each atom follows the equations of MD while the nanotube undergoes axial elongation. We now use this to find the relation between the trajectories of atom $k$ in mole- cule $i$ and the corresponding atom $k$ in molecule 0 . The isometry that maps molecule 0 to molecule $i$ is given by $h_{2}^{i}=\left(\boldsymbol{R}_{\theta_{2}}^{i} \mid i k_{2} \boldsymbol{e}\right)$, where we recall that the nanotube axis $\boldsymbol{e}$ is the same as the axis of the rotation $\boldsymbol{R}_{\theta_{2}}$. Thus,

$$
\begin{aligned}
\boldsymbol{x}_{i, k}(t) & =h^{i}\left(\boldsymbol{x}_{0, k}(t)\right)=\boldsymbol{R}_{\theta_{2}}^{i} \boldsymbol{x}_{0, k}(t)+(a t+b) \boldsymbol{e} \\
& \Rightarrow \dot{\boldsymbol{x}}_{i, k}(t)=\boldsymbol{R}_{\theta_{2}}^{i} \dot{\boldsymbol{x}}_{0, k}(t)+a \boldsymbol{e} \\
& \Rightarrow \ddot{\boldsymbol{x}}_{i, k}(t)=\boldsymbol{R}_{\theta_{2}}^{i} \ddot{\boldsymbol{x}}_{0, k}(t) .
\end{aligned}
$$

For convenience, we define $g_{i}:=\left(\boldsymbol{Q}_{i} \mid \boldsymbol{c}_{i}\right)$ to represent $h_{2}^{i}$. Then, we have from above that $\ddot{x}_{i, k}(t)=\boldsymbol{Q}_{i} \ddot{x}_{0, k}(t)$.

The potential energy $\phi\left(\left\{\boldsymbol{x}_{i, k}\right\}\right): \mathbb{R}^{6 M N} \rightarrow \mathbb{R}$ exhibits two fundamental invariances. The first is frame-indifference: $\phi\left(\left\{\boldsymbol{x}_{i, k}\right\}\right)=\phi\left(\left\{\boldsymbol{Q} \boldsymbol{x}_{i, k}+\boldsymbol{c}\right\}\right)$ for all $\boldsymbol{Q}$ orthogonal and $\boldsymbol{c}$. The second is permutation invariance, i.e., the labeling of the atoms is not important. These properties enable a simple proof that in OS, even with the isometry group being an affine function of time, every atom satisfies the equations of MD. ${ }^{8}$ In addition, the force on an image atom in molecule $i$ is related to the force on the atom in the FD through

$$
\boldsymbol{F}_{i, k}:=-\frac{\partial \phi}{\partial \boldsymbol{x}_{i, k}}=-\boldsymbol{Q}_{i} \frac{\partial \phi}{\partial \boldsymbol{x}_{0, k}}=: \boldsymbol{Q}_{i} \boldsymbol{F}_{0, k}
$$

using the chain rule.

We now consider the effect of adding barostats or thermostats in order to maintain a given temperature or axial force, following the ideas for periodic MD. ${ }^{24}$ We note that time-dependent isometry groups correspond to non-equilibrium settings, whereas thermostats and barostats are equilibrium ideas. Thus, in what follows, we restrict the group elements to be constant in time; barostats and thermostats provide the desired initial conditions for the non-equilibrium calculations.

For isothermal and isobaric simulations, Berendsen ${ }^{24}$ coupled the system to external baths with a fixed reference temperature $\tilde{T}$ and a fixed reference stress tensor $\tilde{\sigma}$. His modified equation of motion is

$$
\begin{aligned}
\dot{\boldsymbol{v}}_{0, k} & =\frac{\boldsymbol{F}_{0, k}}{m_{k}}+\gamma\left(\frac{\tilde{T}}{T}-1\right) \dot{\boldsymbol{x}}_{0, k}, \\
\dot{\boldsymbol{x}}_{0, k} & =\boldsymbol{v}_{0, k}+\beta(\tilde{\sigma}-\sigma) \cdot \boldsymbol{x}_{0, k},
\end{aligned}
$$

where $\sigma$ is the virial stress tensor, $T$ is the averaged temperature, and the scaling constants $\beta$ and $\gamma$ represent the coupling of pressure and thermal baths to the system. The velocity of the $k$ atom in the FD is denoted as $\boldsymbol{v}_{0, k}$. We modify the second line of Eq. (8) to specify the axial force in the $\boldsymbol{e}$ direction:

$$
\begin{aligned}
\dot{\boldsymbol{x}}_{0, k} & =\boldsymbol{v}_{0, k}+\beta(\tilde{\boldsymbol{R}}-\boldsymbol{R}) \cdot \boldsymbol{x}_{0, k}, \text { where } \\
\boldsymbol{R} & =\operatorname{Re} \otimes \boldsymbol{e}, \tilde{\boldsymbol{R}}=\tilde{\boldsymbol{R}} \boldsymbol{e} \otimes \boldsymbol{e},
\end{aligned}
$$

in which $\tilde{R}$ is the specified axial force and $R$ is the virial force in the $z$ direction.

The virial force is work-conjugate to the specific length of the nanotube, and we obtain it from the derivative of the free energy with respect to length in the Appendix. This is similar to the idea behind virial pressure ${ }^{25}$ and virial stress. ${ }^{26}$ The virial force $R$ is averaged over time/ensemble and FD: 


$$
\begin{aligned}
R= & \frac{-1}{L}\left\langle\frac{1}{2} \sum_{i=-N}^{N-1} \sum_{k=1}^{M} \sum_{\substack{j=-N \\
(j, l) \neq(i, k)}}^{N-1} \sum_{l=1}^{M} F_{(i, k)(j, l)} x_{(i, k)(j, l)}\right. \\
& \left.+\frac{1}{3} \sum_{i=-N}^{N-1} \sum_{k=1}^{M} m_{k} \boldsymbol{v}_{i, k} \cdot \boldsymbol{v}_{i, k}\right\rangle
\end{aligned}
$$

where $\langle\ldots\rangle$ represents time-averaging, $L$ is the length of the system in the axial direction, and $\boldsymbol{v}_{i, k}$ is the velocity of the $(i, k)$ atom. The axial components of the force and separation vectors between atoms $(i, k)$ and $(j, l)$ are $F_{(i, k)(j, l)}$ and $x_{(i, k)(j, l)}$, respectively. Using the fact that images of the FD see the same environment up to translation and rotation, this can be simplified to

$$
\begin{aligned}
R= & \frac{-1}{L_{0}}\left\langle\frac{1}{2} \sum_{j=-N^{\prime}}^{N^{\prime}} \sum_{\substack{k=1 \\
(j, l) \neq(0, k)}}^{M} \sum_{l=1}^{M} F_{(0, k)(j, l)} x_{(0, k)(j, l)}\right. \\
& \left.+\frac{1}{3} \sum_{k=1}^{M} m_{k} \boldsymbol{v}_{0, k} \cdot \boldsymbol{v}_{0, k}\right\rangle
\end{aligned}
$$

Here, $L_{0}$ is the axial length of the FD, and atoms in the FD interact with their images as far as the molecules $\pm N^{\prime}$. We have not considered long-range forces such as those due to electromagnetic phenomena.

Now, we show that the modified equations of motion $(8)_{1}$ and (9) hold for all atoms. For atoms in the image cells,

$$
\begin{aligned}
\dot{\boldsymbol{v}}_{i, k} & =\boldsymbol{Q}_{i} \cdot \dot{\boldsymbol{v}}_{0, k}=\boldsymbol{Q}_{i} \cdot \frac{\boldsymbol{F}_{0, k}}{m_{k}}+\gamma\left(\frac{\widetilde{T}}{T}-1\right) \boldsymbol{Q}_{i} \cdot \dot{\boldsymbol{x}}_{0, k} \\
& =\frac{\boldsymbol{F}_{i, k}}{m_{k}}+\gamma\left(\frac{\widetilde{T}}{T}-1\right) \dot{\boldsymbol{x}}_{i, k} .
\end{aligned}
$$

Similarly, we can write the analog of Eq. (9) for atom $(i, k)$ :

$$
\begin{aligned}
\dot{\boldsymbol{x}}_{i, k} & =\boldsymbol{Q}_{i} \cdot \dot{\boldsymbol{x}}_{0, k}=\boldsymbol{Q}_{i} \cdot \boldsymbol{v}_{0, k}+\beta \boldsymbol{Q}_{i} \cdot(\widetilde{\boldsymbol{R}}-\boldsymbol{R}) \cdot \boldsymbol{x}_{0, k} \\
& =\boldsymbol{Q}_{i} \cdot \boldsymbol{v}_{0, k}+\beta \boldsymbol{Q}_{i} \cdot(\widetilde{\boldsymbol{R}}-\boldsymbol{R}) \cdot \boldsymbol{Q}_{i}^{T} \boldsymbol{Q}_{i} \cdot \boldsymbol{x}_{0, k} .
\end{aligned}
$$

Using $\widetilde{\boldsymbol{R}}-\boldsymbol{R}=(\widetilde{R}-R) \boldsymbol{e} \otimes \boldsymbol{e}$, where $\boldsymbol{e}$ is the axis of the nanotube and of the orthogonal tensor $\boldsymbol{Q}$, we have $\boldsymbol{Q}_{i}$. $(\widetilde{\boldsymbol{R}}-\boldsymbol{R}) \cdot \boldsymbol{Q}_{i}^{T}=\widetilde{\boldsymbol{R}}-\boldsymbol{R}$. Thus,

$$
\begin{aligned}
\dot{\boldsymbol{x}}_{i, k} & =\boldsymbol{Q}_{i} \cdot \boldsymbol{v}_{0, k}+\beta(\widetilde{\boldsymbol{R}}-\boldsymbol{R}) \cdot\left(\boldsymbol{Q}_{i} \cdot \boldsymbol{x}_{0, k}+\boldsymbol{b}_{i}-\boldsymbol{b}_{i}\right) \\
& =\boldsymbol{v}_{i, k}+\beta(\widetilde{\boldsymbol{R}}-\boldsymbol{R}) \cdot\left(\boldsymbol{x}_{i, k}-\boldsymbol{b}_{i}\right)
\end{aligned}
$$

where $\boldsymbol{b}_{i}$ is the translation vector of the $i$ th group generator, $g_{i}$ [Eq. (6)]. Comparing Eqs. (12) and (14) with Eqs. (8) and (9), we conclude that all atoms satisfy the modified equation of motion.

The velocity-Verlet time integration of the modified equations is

$$
\boldsymbol{v}_{0, k}\left(t+\frac{\Delta t}{2}\right)=\boldsymbol{v}_{0, k}(t)+\left[\frac{\boldsymbol{F}_{0, k}(t)}{m_{k}}+\gamma\left(\frac{\widetilde{T}}{T(t)}-1\right) \boldsymbol{v}_{0, k}(t)\right] \frac{\Delta t}{2},
$$

$$
\begin{aligned}
\boldsymbol{x}_{0, k}(t+\Delta t)= & {[1+\beta \Delta t(\widetilde{\boldsymbol{R}}-\boldsymbol{R})] \cdot \boldsymbol{x}_{0, k}(t)+\boldsymbol{v}_{0, k}\left(t+\frac{\Delta t}{2}\right) \Delta t } \\
\boldsymbol{v}_{0, k}(t+\Delta t)= & \frac{2}{2-\gamma\left(\frac{\tilde{T}}{T\left(t+\frac{\Delta t}{2}\right)-1}\right) \Delta t} \\
& \times\left[\boldsymbol{v}_{0, k}\left(t+\frac{\Delta t}{2}\right)+\frac{\boldsymbol{F}_{0, k}(t+\Delta t)}{m_{k}} \frac{\Delta t}{2}\right]
\end{aligned}
$$

\section{NON-EQUILIBRIUM OBJECTIVE MOLECULAR DYNAMICS OF SINGLE-WALLED CARBON NANOTUBES}

In this section, we report the results of extensive nonequilibrium objective molecular dynamics calculations that examine the effect of temperature, strain rate, chirality, and pre-stretch/pre-compression, with particular focus on the failure modes. We used the Tersoff potential ${ }^{11}$ to model the atomic interactions. The FD is constructed using subgroups as described above, and we verify our calculations by testing with different choices of FD.

The procedure that we use for all of our calculations is as follows. First, we use the temperature and axial force control as formulated above to relax the nanotubes to the prescribed initial temperature and initial axial force. This is done in order to reach an equilibrium configuration, and no external strain rate is applied during this phase. Rather, the length of the nanotube evolves under the barostat. Next, we remove both the thermostat and the barostat, and a constant strain rate is applied. The system evolves under the influence of the external strain rate. As we update the atomic positions following the Verlet algorithm, we also update the group elements as they change due to the imposed strain rate. This in turn changes the positions of the image atoms that are generated under the action of the group elements. Consequently, the calculation of force on atoms in the FD is coupled to the imposed strain rate.

\section{A. Effect of temperature, strain rate, and chirality}

Figure 2(a) shows failure strain surfaces as a function of chirality and strain rate, with different surfaces corresponding to different initial temperatures. A general trend that we notice is that chirality plays a stronger role at higher strain rates, and its influence decreases at the lower strain rates. We also notice that the failure strain at temperatures of $1500 \mathrm{~K}$ and $1000 \mathrm{~K}$ is extremely small $(\sim 0.08 \%)$ at strain rates of $10^{5}$ to $10^{6} \mathrm{~s}^{-1}$, independent of the chirality. Strain rates below $10^{5} \mathrm{~s}^{-1}$ require extremely long computational times on the order of several months, even with high-performance computing, and thus we do not have comprehensive data for these strain-rate regimes.

However, a limited set of calculations with $(6,6)$ nanotubes shows that the failure strains begin to trend back upward at $10^{4} \mathrm{~s}^{-1}$ [Fig. 2(b)]. While the calculations at $10^{3} \mathrm{~s}^{-1}$ are still in progress, they show that the failure strain is bounded below by $\sim 1 \%$. Because the chirality does not strongly influence the behavior at the lower strain rates, we conjecture that this behavior is shared by other nanotubes. A number of previous studies at higher strain rates $8,15,27-31$ 

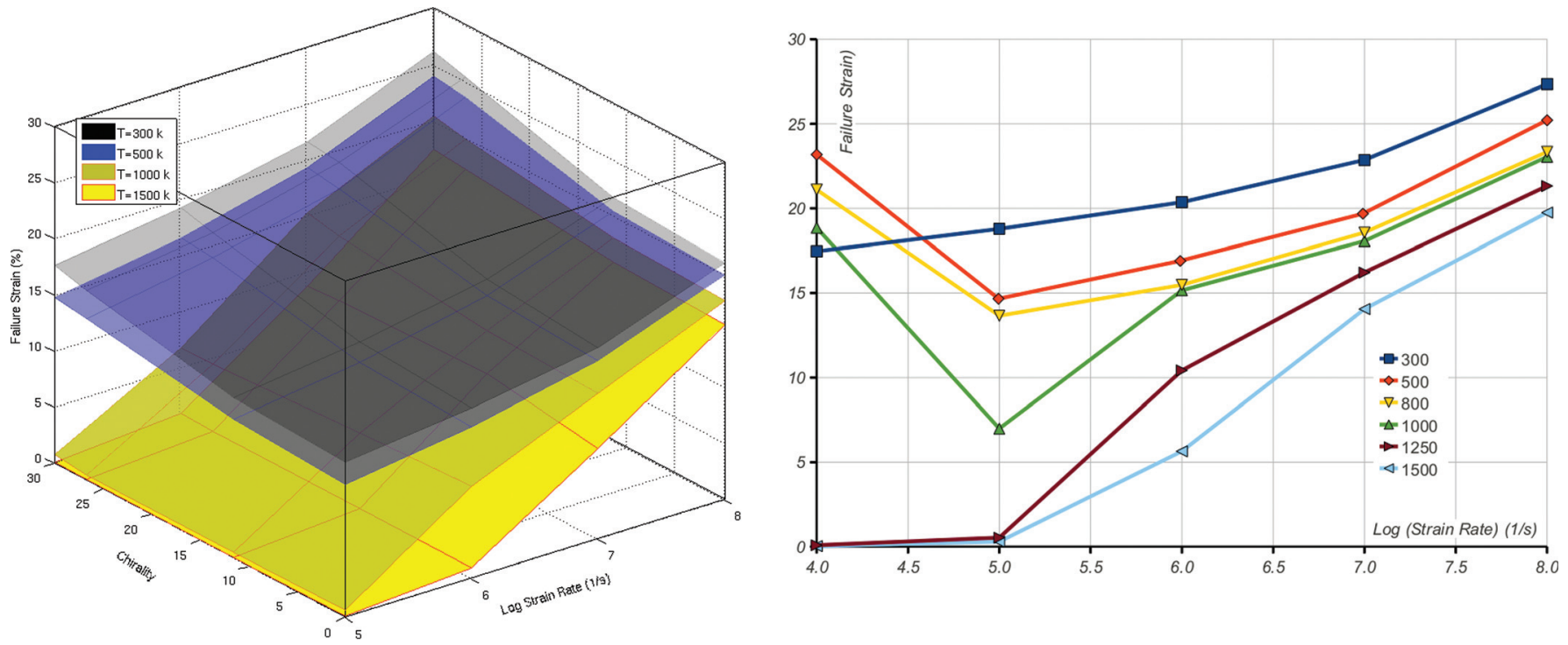

FIG. 2. (Color online) Left: failure strain surfaces as a function of chirality and strain rate, with different surfaces corresponding to different initial temperatures. Right: failure strain for $(6,6)$ nanotubes down to $10^{4} \mathrm{~s}^{-1}$.

have found that reducing the strain rate lowers the failure strain, in contrast to the general expectation that lower strain rates and higher temperatures will promote ductile behavior. Although we also observe this trend, at the lowest rates that we have calculated, this trend begins to reverse.

We observe three primary failure modes of the nanotubes. The mostly widely observed mode was the formation of Stone-Wales defects, in which a bond rotates within the surface of the nanotube, and subsequently two pentagons and two heptagons are created [Fig. 3(a)], leading to a dislocation dipole. Although the Stone-Wales defect can lower the energy significantly, the high barrier for nucleation can require thermal activation, and thus its formation can be temperature-dependent as well as strain-rate dependent. This mode of failure typically correlates with large plastic deformation or ductility and higher failure strains (Fig. 2).

The second widely observed mode can be considered as a microscopic version of brittle fracture. Essentially, the stretching of bonds leads to the breakage of a single bond, which is typically quickly followed by the failure of neighboring bonds [Fig. 3(b)].

The third failure mode is the out-of-surface motion of atoms [Fig. 3(c)]. Bonds rotate out of the plane, and the atom that has moved off the surface bonds with other atoms. (a)

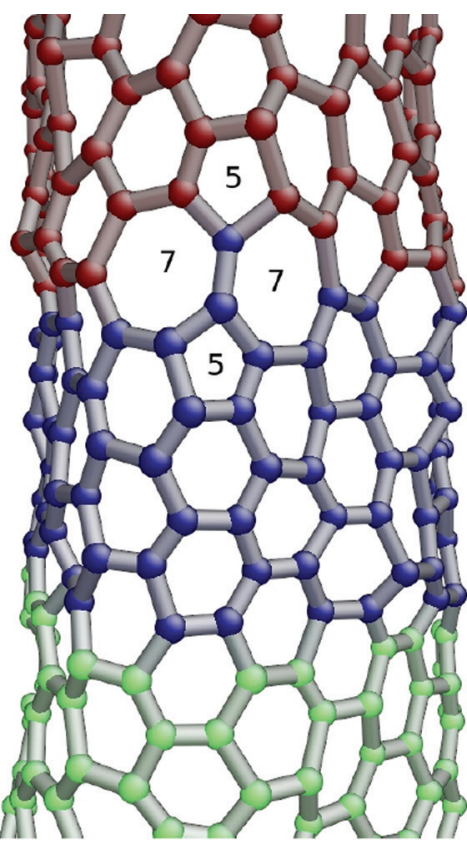

(b)

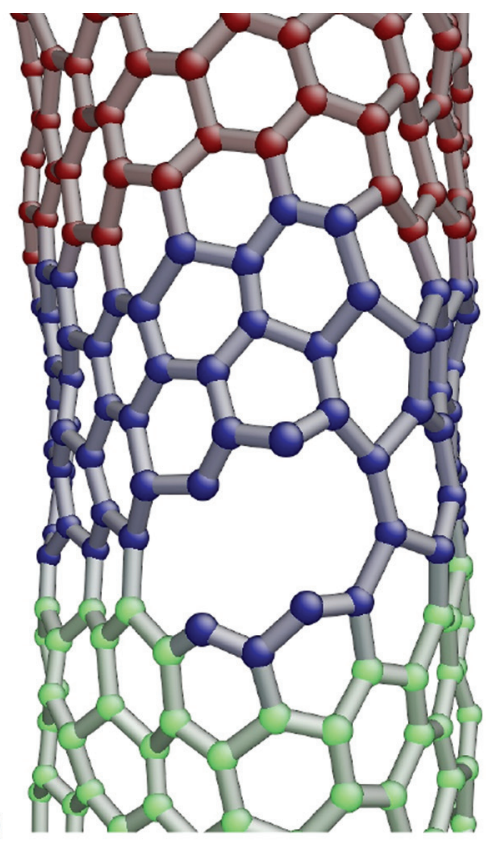

(c)

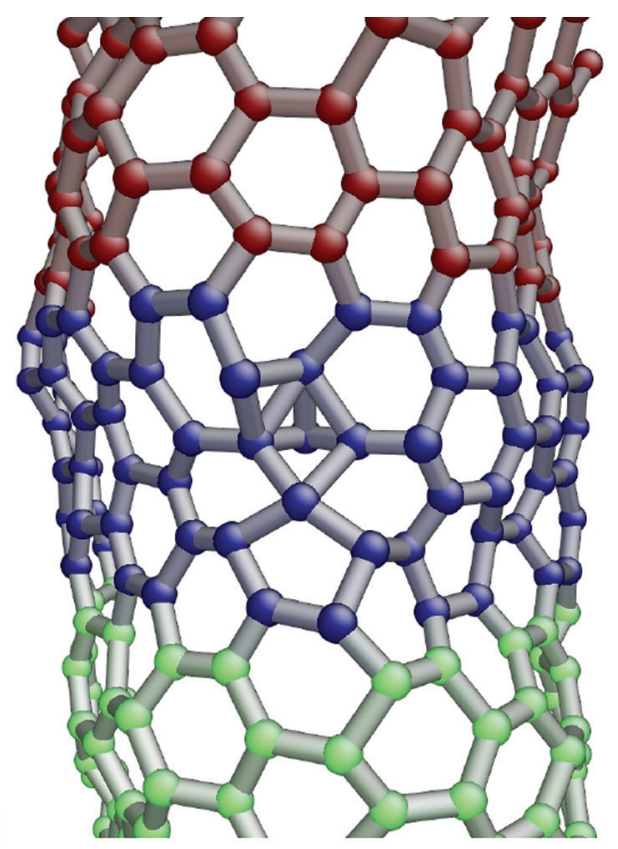

FIG. 3. (Color online) Failure modes: (a) Stone-Wales defect, (b) brittle void formation, and (c) out-of-surface motion. Dark atoms (blue in online color) indicates the simulated atoms in the FD, and other atoms are the images. For clarity, only the atoms on the front surface of the nanotubes are shown. 
(a)
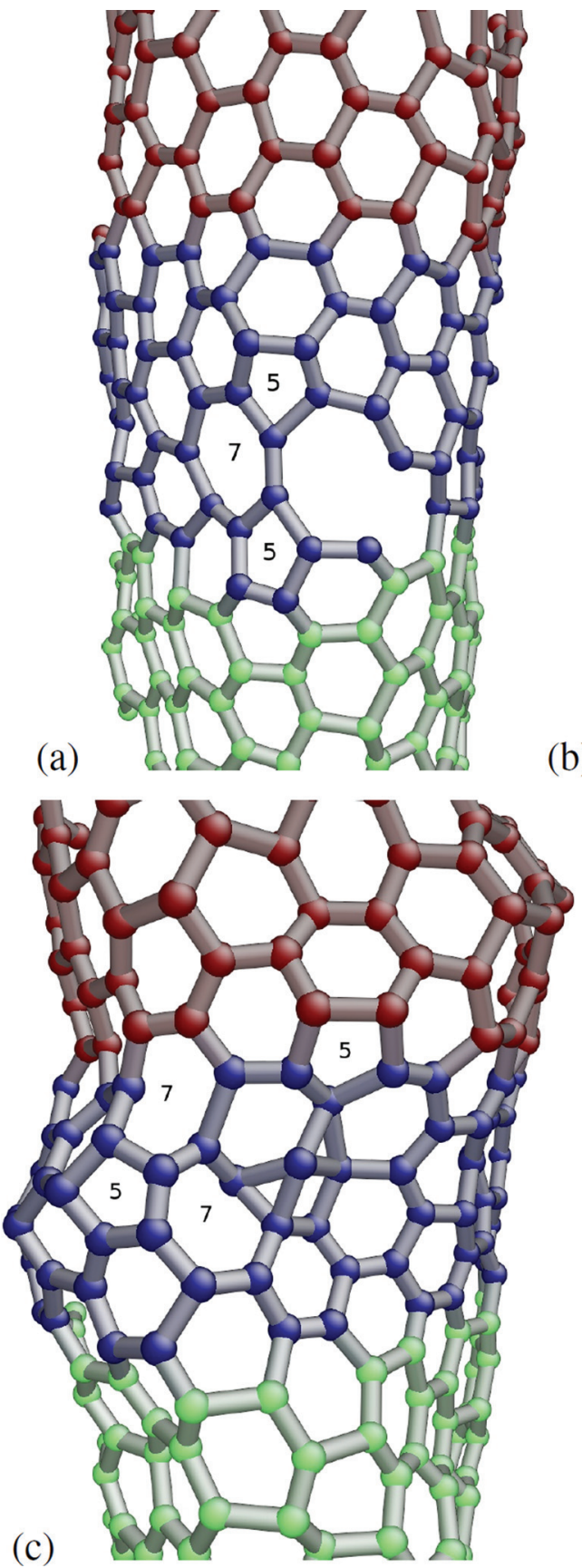

(b)
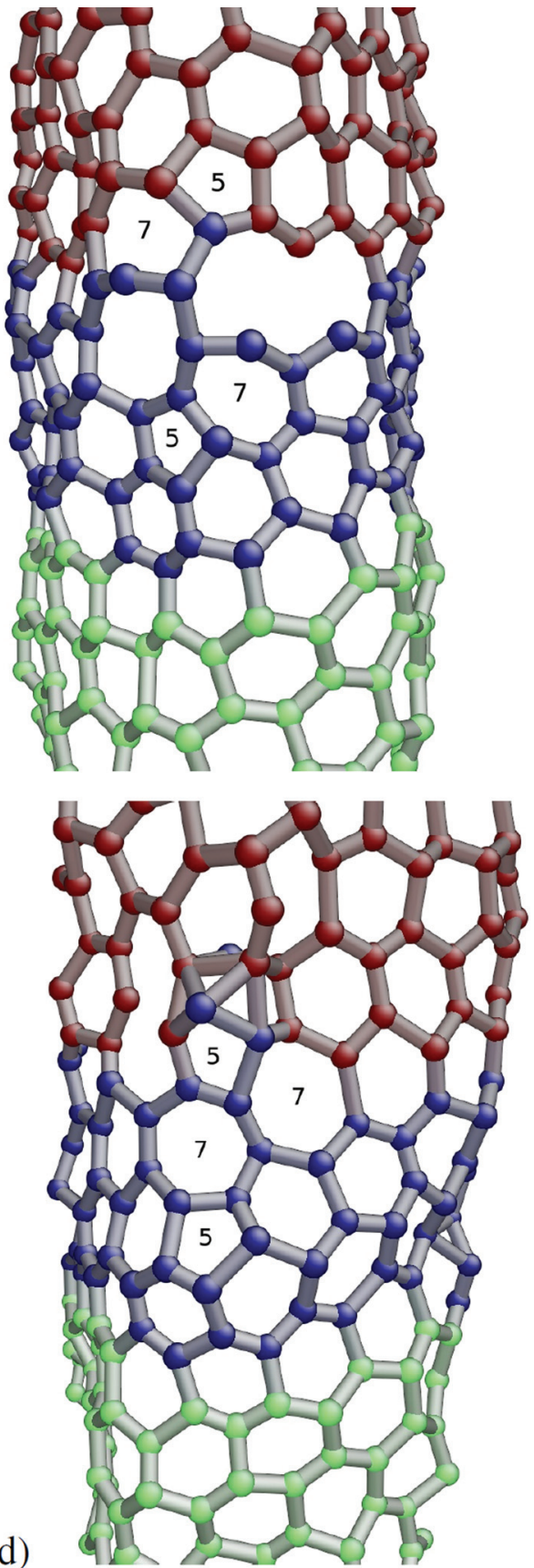

FIG. 4. (Color online) Concurrent failure modes: (a) Stone-Wales and brittle void formation, (b) disassociated StoneWales and two brittle void formations, (c) Stone-Wales and out-of-surface bond rotation, and (d) all three modes.
The failure mechanisms listed above refer to the initial stages of defect formation and growth. In the advanced stages, these mechanisms can be concurrent. For instance, an increasing number of Stone-Wales defects can lead to void formation and subsequent failure in a brittle sense; this is typical of the high failure strain calculations. Figure 4 shows some examples of these mechanisms operating in combination.

We have closely monitored the failure mechanisms of simulations with different temperatures, strain rates, and chiralities, and the results are summarized here.

We find that the out-of-plane bond rotation is the major cause of failure at high temperature (1250-1500 K) and low strain rate $\left(10^{5}\right.$ to $\left.10^{6} \mathrm{~s}^{-1}\right)$. From Fig. 2, nanotubes at these conditions fail at very low strain. Although we observed Stone-Wales defects prior to failure in a few of these calculations, they do not lead to failure, which is instead driven by out-of-surface motion. In the other high temperature calculations (above $1000 \mathrm{~K}$ ), Stone-Wales activity is the primary mechanism of failure. Possibly, this is due to the higher temperature enabling passage of the Stone-Wales activation barrier.

In most cases, at lower temperatures $(300 \mathrm{~K}$ and $500 \mathrm{~K}$ ), we observed Stone-Wales defects prior to failure. However, at the higher strain rates calculated $\left(10^{7} \mathrm{~s}^{-1}\right.$ and $10^{8} \mathrm{~s}^{-1}$ ), brittle fracture occurs before Stone-Wales defects and plays a more significant role in failure. At lower strain rates $\left(10^{5} \mathrm{~s}^{-1}\right)$, Stone-Wales defects usually nucleate before 

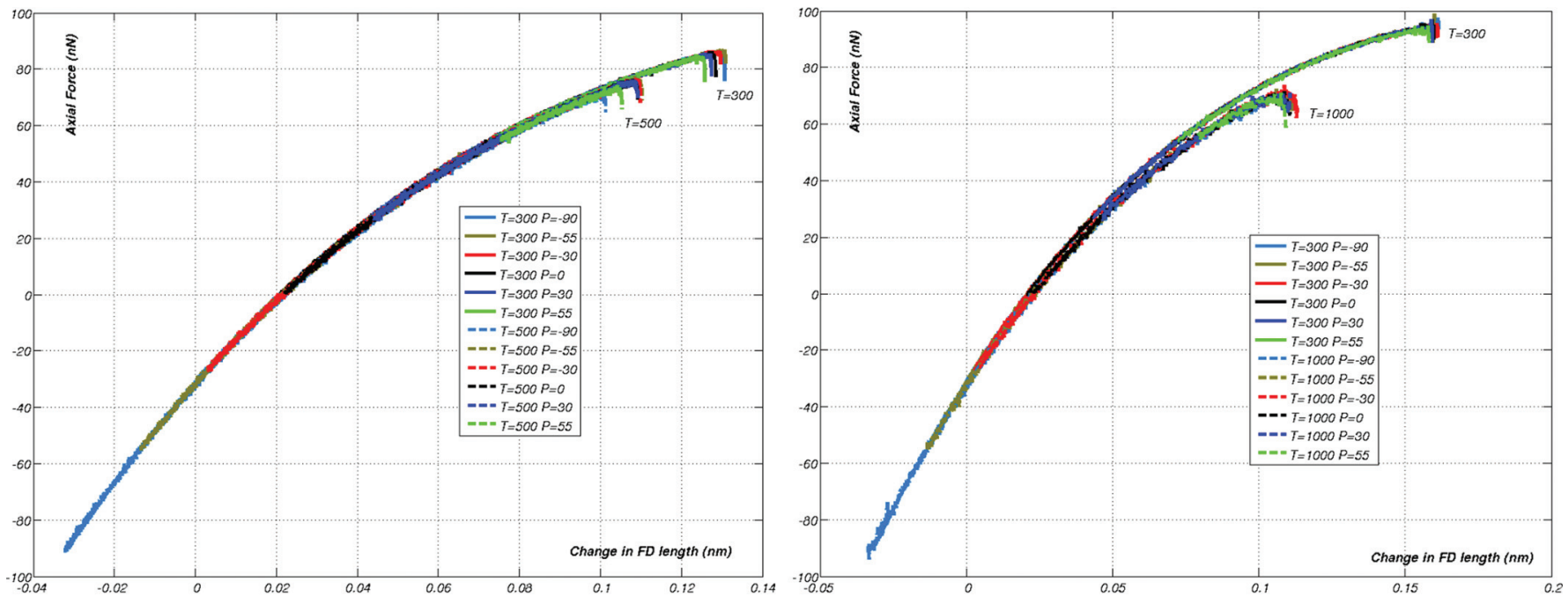

FIG. 5. (Color online) Left: Force-extension curves at $300 \mathrm{~K}$ and $500 \mathrm{~K}$ at a strain rate of $10^{5} \mathrm{~s}^{-1}$ with different axial forces (in nN). Right: Force-extension curves at $300 \mathrm{~K}$ and $1000 \mathrm{~K}$ at a strain rate of $10^{7} \mathrm{~s}^{-1}$ with different axial forces (in $\mathrm{nN}$ ).

brittle fracture, and they play a significant role in failure. For instance, in some simulations, we observed multiple StoneWales defects simultaneously. We also observed that the Stone-Wales defects caused the opening and enlargement of voids. Interestingly, at intermediate strain rates $\left(10^{6} \mathrm{~s}^{-1}\right)$, Stone-Wales defects were infrequently observed. Instead, brittle fracture and consequent void opening were more commonly observed and played the key role in failure. In general, however, the trend was that Stone-Wales defects were observed more often at lower strain rates, possibly because the lower strain rates allow more time for the crossing of the Stone-Wales formation barrier.

Finally, we observe that chirality does not significantly change the failure mechanism but does influence the failure strain. When Stone-Wales defects or brittle fractures are the major cause of the failure, nanotubes with larger chiral angles fail at higher strain. However, when out-of-surface bond rotation is the dominant failure mechanism, there is little change in the failure strain with changes in chirality. These out-of-surface bond rotation cases correspond to high temperature and low strain rate.

\section{B. Effect of pre-stretch and pre-compression}

In this section, we examine the effect of imposing an initial axial force $(-90 \mathrm{nN}$ to $+55 \mathrm{nN})$ at different initial temperatures and strain rates. We note that the structure constructed by Eq. (3) with the values of $\kappa_{2}$ and $l_{0}$ listed there corresponds to a nanotube that is axially compressed by a force of $30 \mathrm{nN}$ at $300 \mathrm{~K}$.

Figure 5 shows the force-extension curves under a range of different conditions (displacement is defined as the change in the length of a FD with a reference length of $0.7379 \mathrm{~nm}$ ). The key finding is that pre-stretch negligibly affects the force-extension behavior and has only a minor role in the final failure. This observation is robust across a range of temperatures, initial stretches, and strain rates. The fact that the nonlinear force-extension behavior is not affected by prestretch can enable the use of axial forces to reliably tune the linearized stiffness of carbon nanotubes, and thereby achieve interesting wave propagation effects. ${ }^{2,32}$ In addition, Fig. 5 also shows the softening of the force-extension curve with temperature.

Figure 6 shows the evolution of temperature for different axial forces with an initial temperature of $300 \mathrm{~K}$. These curves show that the temperature evolution is independent of the initial axial force to within a few degrees Kelvin; additionally, some of the discrepancy in the temperature evolution is due to the fact that our thermostat is set to provide the same initial temperature irrespective of the starting strain. We notice a very sharp increase in the temperature close to the failure strain.

In a previous work, ${ }^{8}$ it was conjectured that extreme cross-sectional distortions seen shortly before failure might be due to the initial axial stress arising from unrelieved thermal expansion. Our formulation of a barostat enables us to test this conjecture, and we find it to be false-the crosssectional distortions are seen with initial compressive and axial forces as well as when the force is absent.

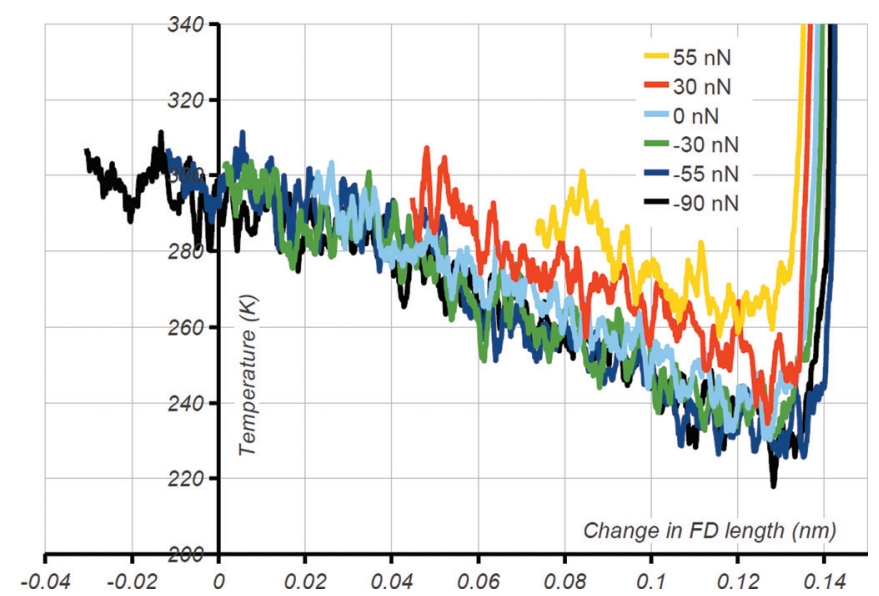

FIG. 6. (Color online) Temperature vs displacement at an initial temperature of $300 \mathrm{~K}$ and strain rate of $10^{6} \mathrm{~s}^{-1}$. 


\section{CONCLUSION}

We have applied the OS framework in order to perform non-equilibrium MD calculations of carbon nanotubes extended at a constant strain rate. We have examined the effect of varying the strain rate from $10^{4}$ to $10^{8} \mathrm{~s}^{-1}$ and the initial temperature from 300 to $1500 \mathrm{~K}$, and we studied a range of chiralities. The OS framework enables the simulation of carbon nanotubes with a small set of atoms, allowing for a larger set of computations at lower strain rates.

We have formulated an analog of a barostat that enables us to exert a specified axial force at equilibrium. The barostat preserves important features of OS: that each atom follows exactly the same equations of motion, and that there are no uncontrolled spurious forces. This enabled us to test the effect of the initial axial force on the failure of carbon nanotubes.

We have found that three modes dominate the failure behavior: first, the formation of Stone-Wales defects; second, the formations of voids and brittle failure; and third, the motion of atoms out of the surface of the nanotube. In addition, these failure modes were also observed to operate concurrently. We have found that both chirality and initial axial stretch/compression play a relatively minor role in comparison with the influence of temperature and strain rate. The OS formulation, in combination with high-performance computing, enables us to access relatively low strain rates (for MD). While our findings at strain rates from $10^{8}$ to $10^{5} \mathrm{~s}^{-1}$ agree qualitatively with those of other studies in that lowering the strain rate reduces the failure strain, we are able to access the regime at $10^{4} \mathrm{~s}^{-1}$. We observe the interesting reversal of this trend: the failure strains at $10^{4} \mathrm{~s}^{-1}$ increase in comparison with those at $10^{5} \mathrm{~s}^{-1}$. Calculations at $10^{3} \mathrm{~s}^{-1}$ are ongoing.

\section{ACKNOWLEDGMENTS}

We thank AFOSR Computational Mathematics (FA9550-09-1-0393) for financial support. This work was supported in part by the National Science Foundation through TeraGrid resources provided by the Pittsburgh Supercomputing Center.

\section{APPENDIX: VIRIAL FORCE FORMULA}

We write an expression for the virial force along the axial direction $\boldsymbol{e}$ of the OS following Ref. 26. The axial force is defined as the derivative of the free energy with respect to the corresponding length dimension $L$. We begin by introducing scaled atomic positions $\left\{\boldsymbol{s}_{i}\right\}$, and the matrix $\boldsymbol{H}$ where $\operatorname{det}(H)=\Omega$ is the volume of the fundamental domain and atomic positions $\boldsymbol{x}_{i}=H \cdot \boldsymbol{s}_{i}$. The free energy of the canonical ensemble is then

$$
\psi=-k_{B} T \ln \left\{\frac{\Omega^{N}}{\Lambda^{3 N} N !} \int d^{3 N} s_{i} \exp \left[-\frac{U\left(\left\{\boldsymbol{s}_{i}\right\}, \boldsymbol{H}\right)}{k_{B} T}\right]\right\},
$$

where $k_{B}$ is Boltzmann's constant, $T$ is the temperature, $\Lambda=\hbar\left(2 \pi m k_{B} T\right)^{-1 / 2}$, and $\hbar$ is Planck's constant. Note that both $\Omega$ and $U$ depend on $L$. Then,

$$
\frac{\partial \psi}{\partial L}=\frac{\Omega^{N} \int \exp \left[-\frac{U\left(\left\{\boldsymbol{s}_{i}\right\}, \boldsymbol{H}\right)}{k_{B} T}\right] \frac{\partial U}{\partial L} d^{3 N} \boldsymbol{s}_{i}-k_{B} T N \Omega^{N-1} \frac{\partial \boldsymbol{\Omega}}{\partial L} \int \exp \left[-\frac{U\left(\left\{\boldsymbol{s}_{i}\right\}, \boldsymbol{H}\right)}{k_{B} T}\right] d^{3 N} \boldsymbol{s}_{i}}{\Omega^{N} \int \exp \left[-\frac{U\left(\left\{\boldsymbol{s}_{i}\right\}, \boldsymbol{H}\right)}{k_{B} T}\right] d^{3 N} \boldsymbol{s}_{i}}
$$

The first and second terms above correspond to the first and second terms, respectively, in Eq. (10).

${ }^{1}$ R. Saito, G. Dresselhaus, and M. S. Dresselhaus, Physical Properties of Carbon Nanotubes (Imperial College Press, London, 1998).

${ }^{2}$ C. Daraio, V. F. Nesterenko, and S. Jin, Appl. Phys. Lett. 85, 5724 (2004).

${ }^{3}$ I. Arias and M. Arroyo, Phys. Rev. Lett. 100(8), 85503 (2008).

${ }^{4}$ M. Arroyo and I. Arias, J. Mech. Phys. Solids 56(4), 1224 (2008).

${ }^{5}$ R. D. James, J. Mech. Phys. Solids 54, 2354 (2006).

${ }^{6}$ I. Nikiforov, D. B. Zhang, R. D. James, and T. Dumitrică, Appl. Phys. Lett. 96, 123107 (2010)

${ }^{7}$ T. Dumitrica and R. D. James, J. Mech. Phys. Solids 55, 2206 (2007).

${ }^{8}$ K. Dayal and R. D. James, J. Mech. Phys. Solids 58, 145 (2010).

${ }^{9}$ W. G. Hoover, Phys. Rev. A 31(3), 1695 (1985).

${ }^{10}$ M. Parrinello and A. Rahman, J. Appl. Phys. 52(12), 7182 (1981).

${ }^{11}$ J. Tersoff, Phys. Rev. Lett. 61(21), 2879 (1988).

${ }^{12}$ B. I. Yakobson, M. P. Campbell, C. J. Brabec, and J. Bernholc, Comput. Mater. Sci. 8(4), 341 (1997).

${ }^{13}$ S. Zhang, S. L. Mielke, R. Khare, D. Troya, R. S. Ruoff, G. C. Schatz, and T. Belytschko, Phys. Rev. B 71(11), 115403 (2005).

${ }^{14}$ C. Wei, K. Cho, and D. Srivastava, Phys. Rev. B 67(11), 115407 (2003).

${ }^{15}$ T. Dumitrica, M. Hua, and B. I. Yakobson, Proc. Natl. Acad. Sci. U.S.A. 103(16), 6105 (2006).

${ }^{16}$ E. Ertekin, D. C. Chrzan, and M. S. Daw, Phys. Rev. B 79(15), 155421 (2009).
${ }^{17}$ Z. Li, P. Dharap, P. Sharma, S. Nagarajaiah, and B. I. Yakobson, J. Appl. Phys. 97, 074303 (2005)

${ }^{18}$ A. J. Stone and D. J. Wales, Chem. Phys. Lett. 128, 501 (1986).

${ }^{19}$ T. Dumitrica, T. Belytschko, and B. I. Yakobson, J. Chem. Phys. 118(21), 9485 (2003)

${ }^{20}$ S. S. Terdalkar, S. Huan, H. Yuan, J. J. Rencis, T. Zhu, and S. Zhang, Chem. Phys. Lett. 494, 218 (2010).

${ }^{21}$ T. Shimada, D. Shirasaki, and T. Kitamura, Phys. Rev. B 81, 035401 (2010).

${ }^{22}$ M. Nardelli, B. I. Yakobson, and J. Bernholc, Phys. Rev. Lett. 81(21), 4656 (1998)

${ }^{23}$ K. Dayal, R. S. Elliott, and R. D. James, Formulas for Objective Structures (to be published).

${ }^{24}$ H. J. C. Berendsen, J. P. M. Postma, W. F. van Gunsteren, A. DiNola, and J. R. Haak, J. Chem. Phys. 81(8), 3684 (1984).

${ }^{25}$ M. J. Louwerse and E. J. Baerends, Chem. Phys. Lett. 421, 138 (2006).

${ }^{26}$ W. Cai, W. Fong, E. Elsen, and C. R. Weinberger, J. Mech. Phys. Solids 56, $3242(2008)$

${ }^{27}$ Y.-R. Jeng, P.-C. Tsai, and T.-H. Fang, Nanotechnology 15, 1737 (2004).

${ }^{28}$ C. Wei, K. Cho, and D. Srivastava, Phys. Rev. B 67, 115407 (2003).

${ }^{29}$ T. Dumitrica and B. I. Yakobson, Appl. Phys. Lett. 84(15), 2775 (2004).

${ }^{30}$ H. Zhao and N. R. Aluru, J. Appl. Phys. 108, 064321 (2010).

${ }^{31}$ X. Liu, D. Cao, and A. Yu, J. Phys. Chem. C 114, 4309 (2010).

${ }^{32}$ C. Daraio, V. F. Nesterenko, S. Jin, W. Wang, and A. M. Rao, J. Appl. Phys. 100, 064309 (2006) 